\title{
Modelling the Inverted-U Effect with ACT-R
}

\author{
Roman V. Belavkin (rvb@Cs.Nott.AC.UK) \\ School of Computer Science and Information Technology, \\ University of Nottingham \\ Jubilee Campus, Wollaton Road, Nottingham NG8 1BB, UK
}

\begin{abstract}
Properties of the ACT-R conflict resolution suggest the possibility of modelling a behaviour at different activation levels of the autonomic nervous system. A model of the classical Yerkes-Dodson experiment was built to test the predictions. The resulting model explains such psychological phenomena as the inverted- $U$ curve, relating performance to arousal, within the mathematical representations of equations in ACT-R. Dynamical optimisation of the two crucial parameters, namely goal value and gain noise variance, may produce more optimal solution paths. We argue that subjects also perform such optimisation as including it into the models can produce a better match with the data. The dynamics corresponds to activational and motivational changes, related to experience of emotion during problem solving, which leads to interesting speculations about a role emotion in intelligence.
\end{abstract}

\section{Introduction}

Recent progress in cognitive modelling has allowed the testing of quite broad range of human psychology and cognition theories. A lot of experiments in psychology have been reproduced by cognitive scientists within theories such as SOAR or ACT. This produced new insights into understanding of some phenomena of human memory, learning, perception and reasoning. Yet there have been few attempts to understand emotion and affect within this framework.

It is known that emotion accompanies problem solving and it is closely related to cognitive processes such as learning, decision making and memory. It is becoming evident that cognitive models should take emotion into account (Belavkin, Ritter \& Elliman 1999). A lot of these models simulate subjects solving various puzzles and problems, some models consider children, as in (Jones, Ritter \& Wood 2000), whose emotions are easily observable. We believe that the cognitive science approach will help us to understand better what happens to the thinking process itself as a result of these emotions. What is the difference between assembling the tower of Hanoi in an angry or a happy mood?

\section{Decision making in ACT-R}

Many ideas in this work were inspired by the results of the Tower of Nottingham model (Jones et al. 2000), which was used to study cognitive development. In particular, the model with increased noise in conflict resolution, which produced a very good match with 7 y.o. children using just the noise variance parameter. This result pointed to the need of a closer investigation into the ACT-R conflict resolution mechanism.

In ACT-R a conflict between several production rules matching the goal is resolved by selecting a rule with the highest expected gain $E=P G-C+\xi(\tau)$, where $P$ is expected probability of achieving the goal if the rule fires, $G$ is the value of the current goal, $C$ is expected cost of that rule, $\xi(\tau)$ is a random variable representing noisy, non-deterministic part of ACT-R, Here $\tau$ is the noise temperature related to the noise variance $\sigma$ as $\tau=\sqrt{6} \sigma / \pi$. In ACT-R $P$ and $C$ can be learned statistically, while $G$ and $\tau$ are set through global parameters.

The probability of selecting a particular $i$-th rule out of $n$ in the conflict set is given by the Boltzmann equation (see eq. 3.4 in Anderson \& Lebiere, 1998). We looked at how this choice probability depends on $P$ s and $C$ s of rules at extreme values of $G$ and $\tau$. The resulting asymptotic properties can be summarised as follows:

i) $\tau \rightarrow 0$ (low noise). The choice is too deterministic: excessive reliance on the past experience, which may become obsolete in a changing environment.

ii) $\tau \rightarrow \infty$ (high noise). The choice is random and it does not depend on the learned expected probabilities $P$ and costs $C$.

iii) $G \rightarrow 0$ (low motivation). The choice is completely determined by $C$ s and not by $P$ s.

iv) $G \rightarrow \infty$ (high motivation). Opposite to the previous, the choice does not depend on $C \mathrm{~s}$, but is purely determined by $P$ s.

The ratio $G / \tau$ determines how much the choice depends on the learned statistics and it may represent confidence. It can be shown that even when 
the ratio remains constant, the costs are more important for low $G$ and $\tau$, while probabilities are more important for high values.

\section{The Yerkes-Dodson experiment model}

Asymptotic properties suggest that together the values of $G$ and $\tau$ represent the activation or the "energy" of a cognitive process (arousal in activation theory of emotion). In order to illustrate different choice strategies at different activation levels, predicted by the properties, a model of the famous Yerkes-Dodson experiment (Yerkes \& Dodson 1908) was built using ACT-R (Fig. 1). One of the objectives was to obtain the Inverted-U curve effect relating performance (speed of learning) and arousal.
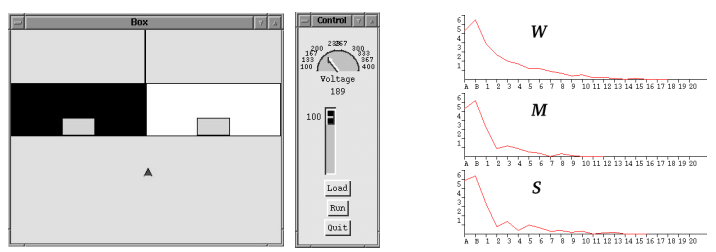

Figure 1: Left: user interface of the Yerkes-Dodson experiment simulation. Right: error curves for weak "W", medium "M" and strong "S" activation. The fastest decay is at medium ("M") stimulation.

The model uses both statistical and procedural learning (production compilation). High noise affects the use of the learned statistics resulting in performance degradation. In changing environment too little noise may result in repetitive errors (observed on the model). At low activation the model is unlikely to make decisions with high costs (as predicted by iii)), but the preference changes with the activation increase.

The model demonstrated that the Inverted-U curve effect can be observed: the fastest learning corresponds to a certain level of activation (values of $G$ and $\tau$ ) even when the ratio $G / \tau$ remains constant.

\section{Optimal performance and emotion}

The optimal activation level $(G, \tau$ values corresponding to the fastest learning) is task specific and it may not be known in advance. If $G$ and $\tau$ remain constant during the simulation run (as they are by default in ACT-R), there is no chance to find their optimal values. The well known optimisation methods, such as simulated annealing, or search methods, such as best-first, suggest the following strategy for dynamic optimisation of $G$ and $\tau$ : problem solving should begin with low goal value $G$ and high noise $\tau$ (high temperature state; also low $G$ results in breadth-first search behaviour); after some statistical information about the application of rules has been learned, the increase of $G$ and decay of $\tau$ will make the choice less random and rely more on the learned information (cooling the system down, or making the search deeper).

This optimisation strategy corresponds well to the behaviour of an emotional problem solver: on experiencing a success motivation and confidence increases (higher $G$ and $G / \tau$ ), as it is accompanied by positive emotions, such as joy. On failure the experienced negative emotion, such as frustration, results in decrease of motivation and confidence $(G$ and $G / \tau)$, which corresponds to heating the system up from a glass state in simulated annealing.

Dynamic control over the goal value $G$ and noise temperature $\tau$ may improve the match between in ACT-R models and data, which we demonstrate on the Tower of Nottingham and Yerkes-Dodson experiment models.

\section{Conclusions}

We showed how some effects of emotions on problem solving can be modelled in cognitive architectures with nondeterministic and statistical learning capabilities, like ACT-R. In such architectures, changes of motivation (maximum cost, or goal value $G$ ) and randomness (noise temperature $\tau$ ) can produce noticeable effects on the behaviour of models. This can be used both for a better data matching and modelling the behaviour of subjects in highly active or passive emotional states. The demonstrated similarity between optimisation methods and emotional reactions during problem solving, suggests that emotion makes a positive contribution to intelligence, as it implements powerful heuristic methods already known and used elsewhere in AI.

\section{Acknowledgements}

This work is sponsored by EPSRC Credit and ORS Award Scheme.

\section{References}

Anderson, J. R. \& Lebiere, C. (1998), The atomic components of thought, Lawrence Erlbaum Associates, Mahwah, NJ, London.

Belavkin, R. V., Ritter, F. E. \& Elliman, D. G. (1999), Towards including simple emotions in a cognitive architecture in order to fit children's behaviour better, in 'Proceedings of the 1999 Conference of the Cognitive Science Society', Lawrence Erlbaum, Mahwah, NJ, p. 784.

Jones, G., Ritter, F. E. \& Wood, D. J. (2000), 'Using a cognitive architecture to examine what develops', Psychological Science 11(2), 93-100.

Yerkes, R. M. \& Dodson, J. D. (1908), 'The relation of strength of stimulus to rapidity of habit formation', Journal of Comparative Neurology and Psychology 18, 459-482. 